\title{
ANFIS and Cost Optimization for Markovian Queue with Operational Vacation
}

\author{
Sonali Thakur \\ Department of Mathematics \& Statistics, \\ Manipal University Jaipur, Rajasthan, 303007, India. \\ E-mails: sonali.thakur010@gmail.com, sonali.191023607@muj.manipal \\ Anamika Jain \\ Department of Mathematics \& Statistics, \\ Manipal University Jaipur, Rajasthan, 303007, India. \\ Corresponding author: anamikajain_02@rediffmail.com \\ Madhu Jain \\ Department of Mathematics, \\ Indian Institute of Technology Roorkee, Roorkee, Uttarakhand-247667, India. \\ E-mail: madhu.jain@ma.iitr.ac.in
}

(Received on October 8, 2020; Accepted on March 5, 2021)

\begin{abstract}
In this paper, we investigate the $\mathrm{M} / \mathrm{M} / 1 / \mathrm{N}$ single server finite capacity Markovian queueing model with operational vacation and impatient behavior of the customers. To recover the server broken down during a busy period, M-threshold recovery policy along with set-up is used. Using the inflow and outflow transition rates, the state probabilities equations for different system states are constructed. For computing the stationary queue length, matrix-geometric analytic is performed. The sensitivity analysis is carried for the validation of the system performance measures. To examine the scope of the adaptive neuro-fuzzy inference system (ANFIS), computational results are presented using matric-geometric and ANFIS approaches.
\end{abstract}

Keywords- M/M/1/N, Operational vacation, Impatient customers, Matrix-geometric method, ANFIS.

\section{Introduction}

The queueing system is a part of our routine life where the customers must wait for the service facility. Practically these situations can be observed in banking sectors, ticket counters, production systems, telecom-network, traffic lights, software systems, etc. Study of this paper is useful to the readers who want to understand about the phenomena of queueing system. In M/M/1/N queueing model with operational vacation, when the system becomes idle, the server randomly moves for operational vacation and after returning from the vacation if the sever still finds the system idle, he again leaves for another vacation. When the server is on vacation, the service may get affected and sometimes may also cause loss of impatient customers. Several authors have studied queueing models with server vacation. Some of the remarkable research works about server vacation in different set up can be notified in the queueing literature. Jain et al. (2016) presented machine repair problems with single server working vacation and controlled arrival of failed machines under Fpolicy. Niranjan et al. (2017) dealt with two service models with the assumptions that service can be provided by the single server if the queue length reaches to a threshold the value 'a', the service is provided in batches if the queue length reaches $c(c>a)$ and server leaves for vacation if the queue 
International Journal of Mathematical, Engineering and Management Sciences

Vol. 6, No. 3, 894-910, 2021

https://doi.org/10.33889/IJMEMS.2021.6.3.053

length is lower down less than ' $a$ '. Ammar (2017) analyzed the transient nature of impatient customers with multiple vacations. Jain et al. (2017) proposed service interruption in $M / M / 1$ model due to functioning vacation along with customers' reneging behavior due to server breakdown. Azhagappan and Deepa (2020) presented model to study the impatient behavior of customers in a markovian queueing situation having provision of working vacation. Tian and Wang (2020) consider $\mathrm{M} / \mathrm{M} / 1$ queue system with the assumption that system states are almost unobservable and fully unobservable and working vacation and multiple vacation.

Practically, the existence of perfect reliable sever is not possible due to the fact that the server may face failure at any instant in time. To recover the server from breakdown state to working state we study the impact of repair setup to overcome the situation. That is very useful for reducing the waiting time of the costumers. There may be many reasons for the server's failure like power supply failures, hardware problems, etc. This interruption in service affects the service rate negatively. The model performed in this paper facilitates valuable insights for reducing the congestion in real-time service system having failure prone server such as in the banking sector, production factories, call centers, etc. To recover the services urgently, repair setups in any form like threshold, backup, restart, etc. are required. In queueing literature, a few researchers considered the server breakdown situations in their studies. Together with the concept of server breakdown and vacation presented by Jeyakumar and Senthilnathan (2012) analyze server breakdown without interruption in an $\mathrm{M}^{\mathrm{x}} / \mathrm{G}(\mathrm{a}, \mathrm{b}) / 1$ queueing system with multiple vacations. Ye and Liu (2017) analyzed Markovian arrival single server queueing model with working breakdown by employing the matrix geometric method to determine the steady state equations. Chang et al., (2018) developed the stationary probabilities by recursive solver algorithm for unreliable-server retrial queue also discuss the feedback of impatient customers. Vijayalakshmi et al. (2018) analyze the finite capacity of Markovian queueing model with working breakdowns and two-phase service by matrix method Ezeagu et al. (2018) examined M/M/1 model with working breakdowns and recovery policies on the basis of k-threshold recovery period and set-up recovery. The concepts of both server breakdown and vacation have been presented by Kalyanaraman and Sundaramoorthy (2019) to study Markovian working vacation queueing model with state-dependent arrival rate and partial breakdown. Chakravarthy et al. (2020) presented a queueing model for a service system with backup server during the absence of the main server. Rao et al. (2017) analyze the two-phase Markovian queueing model where the first phase of service is for all waiting customers in batch mode and second phase of service is in individual mode.

Many authors applied the matrix geometric method for the queueing models and by using this a concrete solution is derived to overcome the problems long queues. By introducing the phase-type probability distribution, Neuts (1981) developed a matrix analysis-based technique that provides an integrated study of multivariate functions. After that many researchers used a matrix geometric approach to find the stability condition and steady state probabilities. Neuts proposed several models and their matrix geometric solutions in his book [Matrix Geometric Solutions in Stochastic Models: An Algorithmic Approach]. Neuts has presented the matrix geometric method for analyzing the equilibrium behavior of M/M/1 type models in detail. Raj and Chandrasekar (2016) studied matrix-geometric method for the queueing model with sever breakdown, phase type service and repair. Ahuja et al. (2019) proposed the continuous-time quasi birth-and-death model by including the optional service and balking. They used matrix geometric (MG) for the analysis purpose and obtained various performance indices.

The implementation of hybrid soft computing based technique artificial neural fuzzy inference 
system (ANFIS) can be used for computing the numerical results for problem not defined precisely. ANFIS architecture has been used by a very few researchers for their works on queueing systems and applications to real life problems. Bhargava and Jain (2014) presented a comparative study of analytical formulae based numerical results and ANFIS results for the unreliable multi server queueing system. Jain and Meena (2018) also considered the hybrid soft computing ANFIS approach to study the performance of machining system. By considering the admission control based on F policy, Sanga and Jain (2019) investigated M/M/1/K queueing system with general retrial times and gave cost optimization and ANFIS results. Sethi et al. (2020) investigated an unreliable M/M/1 queueing system by including the ANFIS computing. Upadhyaya and Kushwaha (2020) presented supplementary variable analysis of $\mathrm{M}^{\mathrm{X}} / \mathrm{G} / 1$ retrial queue with impatient customers, unreliable server, modified vacation policy, delayed repair and Bernoulli feedback. They also used ANFIS computing approach to compare the numerical results obtained by explicit analytical formulae.

In the present article, we focus to develop $\mathrm{M} / \mathrm{M} / 1 / \mathrm{N}$ single server finite capacity queueing model with operational vacation. The concepts of server breakdown and repair setup are also taken into account. The customers are assumed to arrive in Poisson fashion with different arrival rates in different server's status. If the server does not find any customer for service, he moves for operational vacation for any secondary task to utilize idle time. After returning from vacation if the server still fails to find any customer, he leaves for another vacation; on the other hand, if the server finds at least one customer, he immediately starts service on first come first served (FCFS) basis.

In real time system, the server may face any interruption during the normal busy period due to many reasons like power cut, overloading, viruses in the system, any fault in a machine, etc. and it significantly affects the service rendered by the server in a queueing system. To deal with such adverse situations, an immediate repair setup is required. To recover the server from a breakdown, a threshold-based recovery policy is used. The quasi birth death (QBD) process using stability transition rates are developed. To optimize the decision parameters of the system, the cost function is framed. ANFIS based results are also obtained for comparing the numerical results obtained by the analytical MGM.

For a systematic presentation of the study, we arrange this paper into different sections. The model description along with mathematical assumptions and transition rate matrix is provided in section 2. The stability condition defined by Neuts (1981) is established in section 3. Matrix- geometric approach is explained in section 4. In sections 5, steady state queue size distribution is established. Section 6 is devoted to derive some performance measures including the expected numbers of customers in the system in terms of system state probabilities, etc. The cost function is formed in terms of various activities and associated cost elements in section 7. A brief description of the ANFIS approach is given in section 8 . Numerical experiment and sensitivity analysis are provided in section 9. The final section 10 is conclusions and devoted to highlighting the noble features and future scope of investigation done.

\section{Model Description}

We consider a single server finite capacity Markovian M/M/1/N queueing system with operational vacation, impatient customer, server breakdown, and repair. This model is applicable in various fields like banking sector, telecommunication, power Supply Corporation, etc. In such systems, the server breakdown is a common phenomenon as such immediate repair along with set up is required. The cost-benefit study can be done by evaluating the performance metrics in particular when the 
impatient behavior of customers is prevalent. It is very necessary to deal with impatient behavior of customer because it can directly affect the system negatively. For a better understanding of the queueing situation, a state transition rate diagram is shown in Figure 1. The following assumptions are made to formulate Markovian M/M/1/N and to perform mathematical analysis.

Customers arrive in the system according to Poisson process with rate $\lambda_{X}$, Due to the unavailability of the server, the customer may join the queue with probability $\xi_{n}$ or balk with the probability $1-\xi_{n}(0 \leq \mathrm{n} \leq \mathrm{N})$. The joining probability of customers and the effective arrival rate during operational vacation respectively are defined as

$$
\xi_{n}=\left\{\begin{array}{ll}
1 & n=0 \\
\xi_{n} & 1 \leq n \leq N-1 \\
0 & n \geq N
\end{array} \quad \lambda_{X, n}= \begin{cases}\lambda_{X} & n=0 \\
\lambda_{X} \xi_{n} & 1 \leq n \leq N .\end{cases}\right.
$$

During the busy state, broken down state and repair state of the server, the customers also arrive according to Poisson process with different arrival rates $\lambda_{Y,} \lambda_{Z}$ and $\lambda_{K}$ respectively.

The service time in the vacation state is exponentially distributed with mean $\mu_{\mathrm{X}}^{-1}$. After joining the queue, the customer waits for a certain time ( $\mathrm{T})$ to get service. The customer in the queue may get impatient and leave the system without getting service. The reneging rate $\left(\theta_{n}\right)$ and service rate during operational vacation respectively, can be defined by

$$
\theta_{n}=\left\{\begin{array}{ll}
0 & n=0 \\
n \theta & 1 \leq n \leq N-1 \\
N \theta & n=N
\end{array} \quad \mu_{X, n}= \begin{cases}\mu_{X} & n=0 \\
\mu_{X}+\theta_{n} & 1 \leq n \leq N .\end{cases}\right.
$$

The service time during the busy state (operational vacation state) is exponentially distributed with rate $\mu_{Y}\left(\mu_{X} \leq \mu_{Y}\right)$.

When the server does not find any customer for service it randomly moves for an operational vacation with the rate ' $\alpha$ '.

The failure of the server during the busy period may arise. We assume that the server may fail in Poisson fashion with rate ' $\beta$ ' and immediately sent for repair; the repair time is also exponentially distributed with rate ' $\gamma$ '.

After repair, the server again starts providing services to the customers and moves to the busy state with rate ' $r$ '.

The server provides the service on a first-come-first-served (FCFS) basis.

Markovian queueing model with an operational vacation can be modelled by two dimensional continuous-time Markov process $\{\mathrm{G}(\mathrm{t}), \mathrm{N}(\mathrm{t}) ; \mathrm{t} \geq 0\}$, where $\mathrm{N}(\mathrm{t})$ is the number of customers in the system at time $t$ and $G(t)$ is the server state at time $t$ with 
International Journal of Mathematical, Engineering and Management Sciences

Vol. 6, No. 3, 894-910, 2021

https://doi.org/10.33889/IJMEMS.2021.6.3.053

$$
G(t)= \begin{cases}X, & \text { operational vacation state } \\ Y, & \text { busy state } \\ Z, & \text { break down state } \\ K, & \text { repair state. }\end{cases}
$$

The state space of Markov Process is

$$
S=\{(G, 0) \cup(i, n) \mid i=X, Y, Z, K ; 1 \leq n \leq N\}
$$

Following steady state probabilities are defined for formulating the model mathematical

- $P_{X, n} \equiv$ Probability of ' $n$ ' no. of customers in the system when the server is in operational vacation state, $n=0,1,2, \ldots, N$.

* $\mathrm{P}_{\mathrm{Y}, \mathrm{n}} \equiv$ Probability of ' $\mathrm{n}$ ' no. of customers in the system when the server is in busy state, $\mathrm{n}=1$, $2, \ldots, \mathrm{N}$.

* $\mathrm{P}_{\mathrm{Z}, \mathrm{n}} \equiv$ Probability of ' $\mathrm{n}$ ' no. of customers in the system when the server is in broken down state, $\mathrm{n}=1,2, \ldots, \mathrm{N}$.

* $\mathrm{P}_{\mathrm{K}, \mathrm{n}} \equiv$ Probability of ' $\mathrm{n}$ ' no. of customers in the system when the server is in repair state, $\mathrm{n}=1$, $2, \ldots, N$.

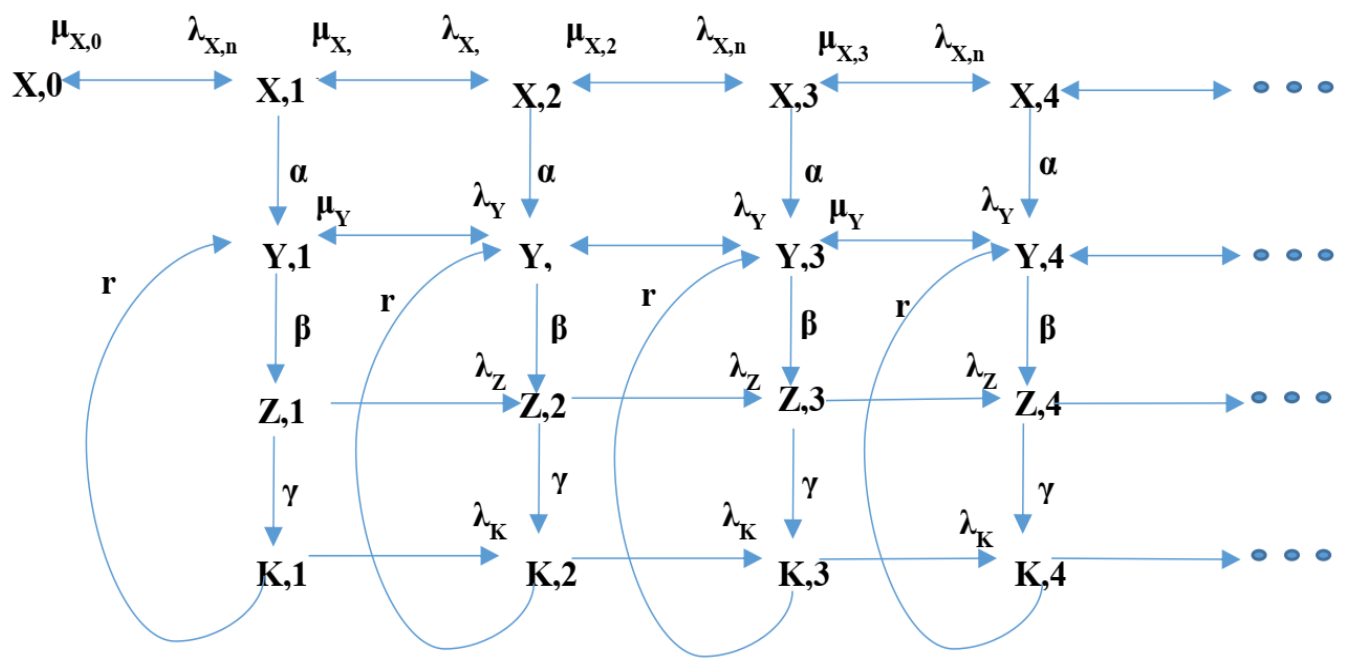

Figure 1. State transition rate diagram.

By using birth- death process, Chapman-Kol-Mogrov differential equations can be formulated as follows: 


\section{- Server is in Operational Vacation Mode}

$$
\begin{aligned}
& \frac{d P_{X, 0}(t)}{d t}=-\lambda_{X, n} P_{X, 0}(t)+\mu_{X, 0} P_{X, 1}(t)+\mu_{Y} P_{Y, 1}(t) \\
& \frac{d P_{X, n}(t)}{d t}=-\left(\mu_{X, n+1}+\lambda_{X, n}+\alpha\right) P_{X, n}(t)+\lambda_{X, n} P_{X, n+1}(t)+\mu_{X, n} P_{X, n+1}(t)
\end{aligned}
$$

- Server is in Busy Mode

$$
\begin{aligned}
& \frac{d P_{Y, 1}(t)}{d t}=-\left(\mu_{Y}+\lambda_{Y}+\beta\right) P_{Y, 1}(t)+\alpha P_{X, 1}(t)+\mu_{Y} P_{Y, 2}(t) \\
& \frac{d P_{Y, n}(t)}{d t}=-\left(\mu_{Y}+\lambda_{Y}+\beta\right) P_{Y, n}(t)+\alpha P_{X, n}(t)+\mu_{Y} P_{Y, n+1}(t)+\lambda_{Y} P_{Y, n-1}(t)
\end{aligned}
$$

- Server is in Broken Down State

$$
\begin{aligned}
& \frac{\mathrm{dP}_{\mathrm{Z}, 1}(\mathrm{t})}{\mathrm{dt}}=-\left(\gamma+\lambda_{\mathrm{Z}}\right) \mathrm{P}_{\mathrm{Z}, 1}(\mathrm{t})+\beta \mathrm{P}_{\mathrm{Y}, 1}(\mathrm{t}) \\
& \frac{d P_{Z, n}(t)}{d t}=-\left(\gamma+\lambda_{Z}\right) P_{Z, n}(t)+\beta P_{Y, n}(t)+\lambda_{Z} P_{Z, n-1}(t)
\end{aligned}
$$

\section{- Server is Under Repair}

$\frac{d P_{K, 1}(t)}{d t}=-\left(r+\lambda_{K}\right) P_{K, 1}(t)+\gamma P_{Z, 1}(t)$

$$
\frac{d P_{K, n}(t)}{d t}=-\left(r+\lambda_{K}\right) P_{K, n}(t)+\gamma P_{Z, n}(t)+\lambda_{K} P_{K, n-1}(t)
$$

\section{a. Transition Rate Matrix}

A matrix analytic approach is employed for determining queue length distribution using the matrixgeometric method and probabilities at stationary state for bi-variate Markov Process.

This method is used to formulate results for the probability vector at the steady- state. The probability vector $\mathrm{P}$ is determined according to generator matrix $\mathrm{Q}$ given by

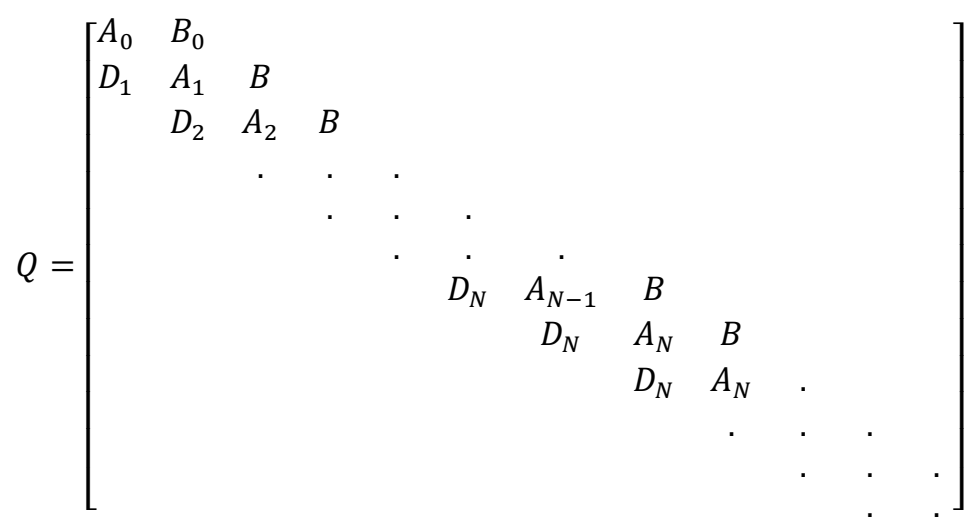


Here $A_{n}, B, D_{n}$ are square matrices of order 4 and are given by where $n=1,2,3, \ldots N$

$$
\begin{aligned}
& A_{0}=\left[-\lambda_{X, n}\right] \quad B_{0}=\left[\begin{array}{llll}
\lambda_{X, n} & 0 & 0 & 0
\end{array}\right] \quad D_{1}=\left[\begin{array}{c}
\mu_{X, N-1} \\
\mu_{Y} \\
0 \\
0
\end{array}\right], \\
& A_{n}=\left[\begin{array}{cccc}
-\left(\lambda_{X, n}+\mu_{X, n-1}+\alpha\right) & \alpha & 0 & 0 \\
0 & -\left(\lambda_{Y}+\mu_{Y}+\beta\right) & \beta & 0 \\
0 & 0 & -\left(\lambda_{Z}+\gamma\right) & \gamma \\
0 & r & 0 & -\left(\lambda_{K}+r\right)
\end{array}\right] \text {, } \\
& B=\left[\begin{array}{cccc}
\lambda_{X, n} & 0 & 0 & 0 \\
0 & \lambda_{Y} & 0 & 0 \\
0 & 0 & \lambda_{Z} & 0 \\
0 & 0 & 0 & \lambda_{K}
\end{array}\right] \quad, \quad D_{n}=\left[\begin{array}{cccc}
\mu_{X, n-1} & 0 & 0 & 0 \\
0 & \mu_{Y} & 0 & 0 \\
0 & 0 & 0 & 0 \\
0 & 0 & 0 & 0
\end{array}\right]
\end{aligned}
$$

and the generator $B+A_{n}+D_{n}$ is equal to

$$
B+A_{n}+D_{n}=\left[\begin{array}{cccc}
-\alpha & \alpha & 0 & 0 \\
0 & -\beta & \beta & 0 \\
0 & 0 & -\gamma & \gamma \\
0 & r & 0 & -r
\end{array}\right]
$$

The quasi birth and death process are represented by this block-tridiagonal generator matrix Q.

Probability vector $\mathbf{P}$ can be partitioned as $\left(\mathrm{P}_{0}, \mathrm{P}_{1}, \mathrm{P}_{2}, \mathrm{P}_{3} \ldots\right)$ where,

$$
\boldsymbol{P}_{\boldsymbol{n}}=\left\{\boldsymbol{P}_{X, \boldsymbol{n}}, \boldsymbol{P}_{Y, \boldsymbol{n}}, \boldsymbol{P}_{\boldsymbol{Z}, \boldsymbol{n}}, \boldsymbol{P}_{\boldsymbol{K}, \boldsymbol{n}}\right\}, \quad \mathrm{n}=0,1,2, .
$$

Now we have $\mathbf{P Q}=\mathbf{0}$ and $\mathbf{P e}=1$; where e is a column vector with entries ' 1 '.

\section{Stability Condition}

Stability condition known as Neuts mean drift condition defined by Neuts (1981) in theorem 6.4 states that Markov process $Q$ is stable if and only if

$\pi B e<\pi D_{n} e$

The equilibrium distribution $\pi$ with generator $E=B+A_{n}+D_{n}$ satisfies the following properties

$\pi E=0 \quad$ And $\quad \pi e=1$

For our model, (9) yield

$\pi \lambda_{Y}+\pi \lambda_{Z}+\pi \lambda_{K}<\pi \mu_{Y}$ 
International Journal of Mathematical, Engineering and Management Sciences

Vol. 6, No. 3, 894-910, 2021

https://doi.org/10.33889/IJMEMS.2021.6.3.053

where $\pi=\left(0, \frac{r}{r+\beta+\gamma}, \frac{\beta}{r+\beta+\gamma}, \frac{\gamma}{r+\beta+\gamma}\right)$.

\section{Matrix Geometric Method}

For Markov process $\mathrm{Q}$, the equilibrium probability vector $\mathrm{P}_{\mathrm{n}}$ has the matrix-geometric form

$P_{n}=P_{1} R^{n-1} \quad \mathrm{n}=1,2,3 \ldots$

where $\mathrm{R}$ is the rate matrix of $\mathrm{Q}$ and is given by

$$
B+R A_{N}+R^{2} D_{N}=0
$$

Following the results of Neuts (1981), we get $R=\lim _{n \rightarrow \infty} R$ where $\left\langle R_{n}\right\rangle$ the sequence is given by

$$
R_{0}=0 \quad \text { and } \quad R_{n+1}=-B A_{N}^{-1}-R^{2} D_{N} A_{N}^{-1} \quad \mathrm{n} \geq 0
$$

\section{Steady State Queue Size Distribution}

Matrix E shows that the equilibrium probability vector $\mathrm{P}$ and $\mathrm{Q}$ exists. By using a recursive relation and matric, we have

$$
\begin{aligned}
& P_{0}=P_{1}(-D) A_{0}^{-1}=P_{1} \Omega_{0} \\
& P_{n-1}=P_{n}\left(-A_{n} B^{-1}-R D_{n} B^{-1}\right)=P_{n} \Omega_{n} \\
& P_{N}=-P_{N-1} B\left(A_{n}+R D_{n}\right)^{-1}
\end{aligned}
$$

\section{Performance Measures}

Some important performance measures of the system are mentioned below:

\subsection{System State Probabilities}

To understand the distribution of customers in the system, the system state probabilities are evaluated as-

- Probability that sever is in a vacation state $\mathrm{P}(\mathrm{X})=\sum_{n=0}^{\infty} P_{X, n}(t)$

- Probability that sever is in a busy state $\mathrm{P}(\mathrm{Y})=\sum_{n=0}^{\infty} P_{Y, n}(t)$

- $\quad$ Probability that sever is in a breakdown state $\mathrm{P}(\mathrm{Z})=\sum_{n=0}^{\infty} P_{Z, n}(t)$

- Probability that sever is in repair state $\mathrm{P}(\mathrm{K})=\sum_{n=0}^{\infty} P_{K, n}(t)$

The probability of $\mathrm{n}$ customers in the system at time $\mathrm{t}>0$ is obtained by

$$
P_{n}(t)=\mathrm{P}(\mathrm{X})+\mathrm{P}(\mathrm{Y})+\mathrm{P}(\mathrm{Z})+\mathrm{P}(\mathrm{K})
$$

\subsection{Expected Numbers of Customers in the System}

An average number of customers waiting in the queue at different state is defined as:

- The average number of customers in the vacation state $\mathrm{E}(\mathrm{X})=\sum_{n=0}^{\infty} n P_{X, n}(t)$ 
- The average number of customers in the busy state $\mathrm{E}(\mathrm{Y})=\sum_{n=0}^{\infty} n P_{Y, n}(t)$

- The average number of customers in the repair state $\mathrm{E}(\mathrm{Z})=\sum_{n=0}^{\infty} n P_{Z, n}(t)$

- The average number of customers in the repair state $\mathrm{E}(\mathrm{K})=\sum_{n=0}^{\infty} n P_{K, n}(t)$

The average of the customers in the system is determined as

$\mathrm{E}(N)=\mathrm{E}(\mathrm{X})+\mathrm{E}(\mathrm{Y})+\mathrm{E}(\mathrm{Z})+\mathrm{E}(\mathrm{K})$

The output is obtained as $O P=\mu_{X, n} \sum_{n=0}^{\infty} P_{X, n}+\mu_{Y} \sum_{n=0}^{\infty} P_{Y, n}$

Average time of waiting $E(W)=\frac{E(N)}{\lambda_{e f f}}$

where $\quad \lambda_{e f f}=\lambda_{X, 0} P_{X, 0}+\sum_{n=1}^{\infty}\left(\lambda_{X, n} P_{X, n}+\lambda_{Y} P_{Y, n}+\lambda_{Z} P_{Z, n}+\lambda_{K} P_{K, n}\right)$.

The average delay time is $\quad E(D)=\frac{E(N)}{O P}$

\section{Cost Model}

In this section, we drive the cost function to make this model economically applicable. The cost model is constructed to find the total cost per unit of time. To build the cost function we consider all chosen parameters with the required recovery rate with the help of the M-threshold repair set up.

We assume that service rate quick reform with $\mathrm{M}$ recovery rate that is required to maintain frequent functioning of queueing system. Following cost elements are introduced for constructing the cost function:

$\mathrm{C}_{\mathrm{h}}$ : holding cost per unit time per customer in the system

$\mathrm{C}_{\mathrm{XX}}$ : cost per unit time when the server is in vacation mode

$\mathrm{C}_{\mathrm{YY}}$ : cost per unit time when the server is in busy mode

$\mathrm{C}_{\mathrm{ZZ}}$ : cost per unit time when the server is in breakdown mode

$\mathrm{C}_{\mathrm{KK}}$ : cost per unit time when the server is in repair mode

$\mathrm{C}_{\mathrm{X}}$ : cost per unit time when the service rate is $\mu_{\mathrm{X}}$

$\mathrm{C}_{\mathrm{Y}}$ : cost per unit time when the service rate is $\mu_{\mathrm{Y}}$

$\mathrm{C}_{\mathrm{K}}$ : cost per unit time when the repair rate is $\mathrm{r}$

On the bases of these parameters expected cost function

$$
\begin{aligned}
& F\left(M, \mu_{X}, \mu_{Y}, r\right)=C_{h} E(N)+C_{X X} P(X)+C_{Y Y} P(Y)+C_{Z Z} P(Z)+C_{Z Z} P(K)+C_{X} \mu_{X}+C_{Y} \mu_{Y}+ \\
& C_{K} r
\end{aligned}
$$

\section{Adaptive Neuro-Fuzzy Inference System (ANFIS) Based Model}

ANFIS modal can be used practically in many areas including transportation, traffic, telecommunication, atmosphere survey, etc. Telecommunication systems involve the artificial 
neural network to achieve various objectives like increase in customers, reduce cost, reduce waiting time, etc. In the present modal we can investigate the impatient tendency of the customer while they are waiting for the service with variation in arrival rate in vacation mode, service rate, repair rate, and repair to busy rate.

A combination of soft computing techniques, artificial neural networks (ANNs), and fuzzy systems (FS) develop a very useful technique for ANFIS. By using the fuzzified parameters we are presenting a brief concept of ANFIS architecture. In the form of fuzzy if-then rules, we can perform an ANFIS input-output function and input-output data pairs. The expressions of the 'IF U AND V THEN', where $U$ and $V$ are fuzzy sets with required membership functions. Three important components of fuzzy inference system are

(i) Selection of fuzzy rules.

(ii) To form a data structure to define the membership functions used in fuzzy rules.

(iii) A reasoning mechanism, which performs the inference procedure based on the given fuzzy rules.

The fuzzy rules can be defined as:

Rule 1: If $u$ is $U_{1}$ and $v$ is $V_{1}$ then $f_{1}=p_{1} u+q_{1} v+r_{1}$.

Rule 2: If $u$ is $U_{2}$ and $v$ is $V_{2}$ then $f_{2}=p_{2} u+q_{2} v+r_{2}$.

Implementation of ANFIS network can be used for comparing the computational results by using the fuzzy toolbox of MATLAB software. In our model neuro-fuzzy model is applied to determine the expected number of customers in the system $\mathrm{E}(\mathrm{N})$ concerning the change in arrival rate in vacation state $(\lambda)$, service rete $(\mu)$, repair rate $(r)$.

The ANFIS architecture with input variable $\gamma$ and $r$ with two-layer network presented in Figure. 2

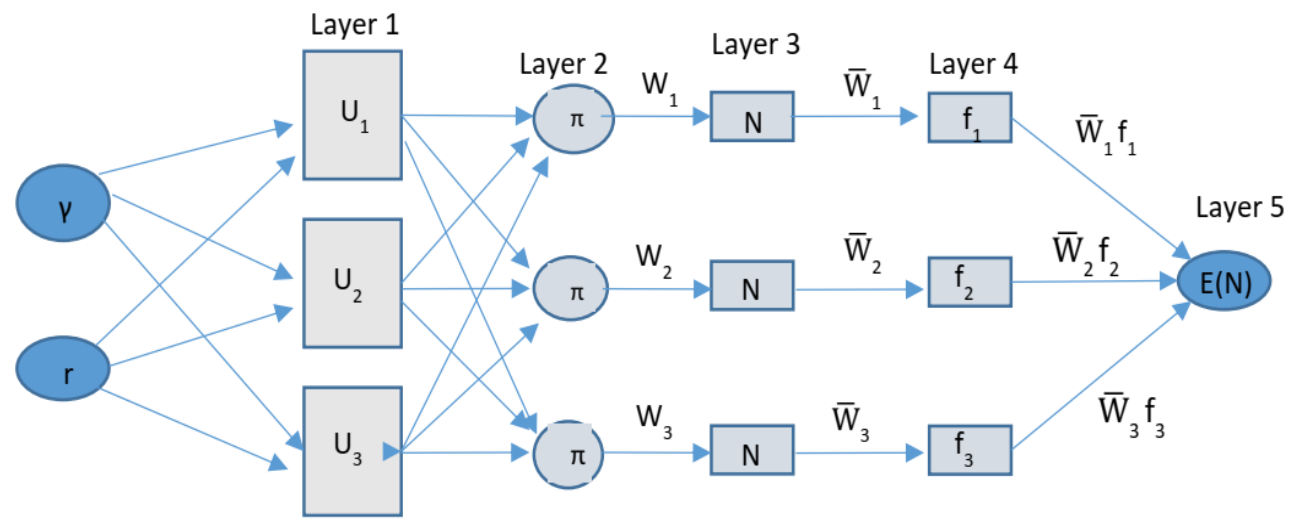

Figure 2. ANFIS architecture.

\section{Numerical Experiment and Sensitivity Analysis}

To explore the sensitivity of the described system model for the various performance measures and to perform the cost analysis, numerical results are presented by developing coding in MATLAB software. The effects of different parameters on performance measures are displayed in Table 1 
International Journal of Mathematical, Engineering and Management Sciences

Vol. 6, No. 3, 894-910, 2021

https://doi.org/10.33889/IJMEMS.2021.6.3.053

and Table 3 and in Figure 3-5.

\subsection{Sensitivity Analysis}

Table 1 reveals the impacts of variation in different parameters on the average number of customers in vacation state $\mathrm{E}(\mathrm{X})$, average number of customers in busy state $\mathrm{E}(\mathrm{Y})$, average number of customers in breakdown state $\mathrm{E}(\mathrm{Z})$, average number of customers in repair state $\mathrm{E}(\mathrm{K})$, output $(\mathrm{OP})$, average waiting time $\mathrm{E}(\mathrm{W})$ and average delay time $\mathrm{T}(\mathrm{D})$ in the system. To perform the numerical experiment, we set $\mathrm{N}=30, \lambda_{X, n}=1.5, \lambda_{Y}=3, \lambda_{Z}=1.5, \lambda_{K}=2.5, \mu_{X}=3, \mu_{Y}=6, \alpha=0.5, \beta=$ $3, \gamma=5, r=3.5$ where $\lambda_{X, n}, \lambda_{Y}, \lambda_{Z}, \lambda_{K}$ are the heterogeneous functions of $\lambda$ described as $\lambda_{X, n}=\lambda$, $\lambda_{Y}=1.2 \lambda, \lambda_{Z}=5 \lambda, \lambda_{K}=8 \lambda$. In Table 1 , variations in different performance measures by varying values of $\lambda$ for different values of $n, r, \mu_{Y}$ are displayed. As expected with the increase in $\lambda, E(W)$ and $\mathrm{T}(\mathrm{D})$ are increasing. But with the increasing the values of $\mathrm{r}$ and $\mu_{Y}$, OP decreasing.

We use the neuro-fuzzy tool for computing the ANFIS results by considering Gaussian membership function for the input parameters $\lambda, \mu_{Y}, \mathrm{r}$, and $\gamma$. In Figure. 3(a-d) we show the fuzzification of $\lambda$, $\mu_{Y}, \mathrm{r}$ and $\gamma$ by considering five members as (i) very low (ii) low (iii) moderate (iv) high (v) very high. This structure is presented in Table 2 whereas the variation via graph in Figure 3(a-d). From Table 3 optimal expected cost TC $=300.4294$ is determined at optimal parameter $\lambda=0.5$ and $\mu_{Y}=$ 7 with effective parameters at $\beta=3$ and $\gamma=5$ it can be useful in real-life aspects.

In Figure 4(a-f), we describe the effect of various parameters on the average queue length $E(N)$ and compare the results with numerical results generated by the neuro-fuzzy results (marked by tick marks) in MATLAB for computing ANFIS model. In Figure 4(a-f), we observe that E(N) increases due to the increase in arrival ( $\lambda$ ) that is quite reasonable. Figure 4(a) and 4(b) present the increasing effect on $\mathrm{E}(\mathrm{N})$ with increasing values of breakdown rate $(\beta)$ and vacation rate $(\alpha)$. But in Figure 4(c) to 4(d) it is observed that $E(N)$ increases by the rise in $r$ and $\gamma$. In Figure 4(e) and 4(f) depicted the decreasing trend in vacation rate $\left(\mu_{X}\right)$ and service rate in busy state $\left(\mu_{Y}\right)$. By using the ANFIS model similar effects are notified in $\mathrm{E}(\mathrm{N})$. We see a very less error in ANFIS results with respect to analytical model results as both graphs almost coincide.

\subsection{Cost Analysis}

To make the model economically beneficial and for future better design of the system, cost analysis of the model is required. The situation of delay in service cannot be avoided as such we would like to determine the system functioning at an optimal and beneficial economic cost. Here, we consider the arrival rate in the busy state $\left(\lambda_{Y}\right)$ and service rate in busy rate $\left(\mu_{Y}\right)$ as decision-making variables. To determine the minimum cost function $\mathrm{F}^{*}$, the variations in the optimal arrival rate in the busy state $\left(\lambda_{Y}^{*}\right)$ and optimal service rate in busy rate $\left(\mu_{Y}^{*}\right)$ are considered in the feasible range 5 to 10 and 0.5 to 2 respectively. We also evaluate the effective optimal breakdown rate $\left(\beta^{*}\right)$ and optimal repair rate $\left(\gamma^{*}\right)$ within the range 3 to 5 and 5 to 7 , respectively. To determine the optimal service rate $\left(\mu_{\mathrm{Y}}^{*}\right)$ and optimal arrival rate $\left(\lambda_{\mathrm{Y}}^{*}\right)$, we consider six sets of cost elements in $\$$ as given below:

Set 1: $C_{h}=5 ; C_{X X}=150 ; C_{Y Y}=200 ; C_{Z Z}=250 ; C_{K K}=200 ; C_{X}=5 ; C_{Y}=10 ; C_{K}=15$

Set 2: $\mathrm{C}_{\mathrm{h}}=3 ; \mathrm{C}_{\mathrm{XX}}=150 ; \mathrm{C}_{\mathrm{YY}}=120 ; \mathrm{C}_{\mathrm{ZZ}}=250 ; \mathrm{C}_{\mathrm{KK}}=150 ; \mathrm{C}_{\mathrm{X}}=5 ; \mathrm{C}_{\mathrm{Y}}=10 ; \mathrm{C}_{\mathrm{K}}=15$

Set 3: $C_{h}=5 ; C_{X X}=200 ; C_{Y Y}=150 ; C_{Z Z}=250 ; C_{K K}=110 ; C_{X}=10 ; C_{Y}=15 ; C_{K}=15$

Set 4: $C_{h}=5 ; C_{X X}=200 ; C_{Y Y}=150 ; C_{Z Z}=250 ; C_{K K}=100 ; C_{X}=10 ; C_{Y}=15 ; C_{K}=20$ 
International Journal of Mathematical, Engineering and Management Sciences

Vol. 6, No. 3, 894-910, 2021

https://doi.org/10.33889/IJMEMS.2021.6.3.053

Set 5: $C_{h}=5 ; C_{X X}=200 ; C_{Y Y}=100 ; C_{Z Z}=200 ; C_{K K}=500 ; C_{X}=20 ; C_{Y}=5 ; C_{K}=15$

Set 6: $C_{h}=3 ; C_{X X}=100 ; C_{Y Y}=50 ; C_{Z Z}=200 ; C_{K K}=500 ; C_{X}=15 ; C_{Y}=10 ; C_{K}=20$.

By plotting the surface graphs for cost function TC $\left(\mu_{Y}^{*}, \lambda_{Y}^{*}\right)$ we make this study more useful for the cost-benefit point of view. The impact of variations in $\lambda$ and $\mu_{\mathrm{Y}}$, on the total cost function TC can be seen in Figure 5(a-f) for all six cost sets.

Table 1. Variation in different system performances by varying $\lambda$ for different values of $r, \mu_{\mathrm{Y}}$.

\begin{tabular}{|c|c|c|c|c|c|c|c|c|}
\hline$\left(\mathbf{n}, \mathbf{r}, \mu_{Y}\right)$ & $\lambda$ & $\mathbf{E}(\mathbf{X})$ & $\mathbf{E}(\mathbf{Y})$ & $\mathbf{E}(\mathbf{Z})$ & $\mathbf{E}(\mathbf{K})$ & OP & $\mathbf{E}(\mathbf{W})$ & $\mathbf{T}(\mathbf{D})$ \\
\hline & 0.5 & 0.24104 & 0.141922 & 0.098468 & 0.156988 & 3.505946 & 0.758731 & 0.182096 \\
\hline & 1 & 0.235249 & 0.204624 & 0.138674 & 0.217685 & 3.421732 & 0.96956 & 0.232699 \\
\hline \multirow[t]{4}{*}{$(30,3.5,6)$} & 1.5 & 0.226659 & 0.321456 & 0.212478 & 0.328306 & 3.296882 & 1.375997 & 0.330281 \\
\hline & 2 & 0.212782 & 0.569525 & 0.366513 & 0.559262 & 3.095635 & 2.296861 & 0.551771 \\
\hline & 2.5 & 0.188138 & 1.166572 & 0.729505 & 1.113862 & 2.74093 & 4.826808 & 1.166785 \\
\hline & 3 & 0.143369 & 2.623924 & 1.592437 & 2.500162 & 2.10901 & 12.86003 & 3.25266 \\
\hline \multirow[t]{6}{*}{$(30,4,6)$} & 0.5 & 0.24362 & 0.12543 & 0.087966 & 0.121877 & 3.543463 & 0.680704 & 0.163369 \\
\hline & 1 & 0.23862 & 0.177073 & 0.1213 & 0.165778 & 3.470742 & 0.843678 & 0.202484 \\
\hline & 1.5 & 0.231351 & 0.270133 & 0.180487 & 0.24315 & 3.365062 & 1.14544 & 0.274919 \\
\hline & 2 & 0.219898 & 0.46067 & 0.299685 & 0.398604 & 3.198777 & 1.795345 & 0.431058 \\
\hline & 2.5 & 0.199938 & 0.910732 & 0.575679 & 0.763032 & 2.910465 & 3.494974 & 0.841577 \\
\hline & 3 & 0.162625 & 2.059451 & 1.262707 & 1.70826 & 2.379639 & 8.856427 & 2.182282 \\
\hline \multirow[t]{6}{*}{$(40,4,7)$} & 0.5 & 0.250044 & 0.081453 & 0.058551 & 0.082263 & 3.636907 & 0.541108 & 0.129866 \\
\hline & 1 & 0.247267 & 0.104714 & 0.073818 & 0.102575 & 3.596509 & 0.612137 & 0.146913 \\
\hline & 1.5 & 0.24362 & 0.140179 & 0.096818 & 0.132939 & 3.543459 & 0.721464 & 0.173151 \\
\hline & 2 & 0.238618 & 0.198314 & 0.134056 & 0.181706 & 3.470713 & 0.903625 & 0.21687 \\
\hline & 2.5 & 0.231339 & 0.303727 & 0.200715 & 0.268318 & 3.36485 & 1.243351 & 0.298408 \\
\hline & 3 & 0.219805 & 0.524206 & 0.338208 & 0.446092 & 3.197201 & 1.991411 & 0.478015 \\
\hline \multirow[t]{6}{*}{$(40,5,7)$} & 0.5 & 0.252248 & 0.071872 & 0.052352 & 0.057889 & 3.668954 & 0.493285 & 0.118388 \\
\hline & 1 & 0.249899 & 0.091051 & 0.065043 & 0.071291 & 3.634789 & 0.547124 & 0.13131 \\
\hline & 1.5 & 0.246858 & 0.119559 & 0.08368 & 0.090847 & 3.590569 & 0.627739 & 0.150657 \\
\hline & 2 & 0.242769 & 0.164717 & 0.112835 & 0.12124 & 3.531088 & 0.757039 & 0.181689 \\
\hline & 2.5 & 0.236975 & 0.242771 & 0.162583 & 0.172753 & 3.44681 & 0.985306 & 0.236474 \\
\hline & 3 & 0.228136 & 0.3956 & 0.258669 & 0.271689 & 3.318288 & 1.449105 & 0.347798 \\
\hline
\end{tabular}

Table 2. The number of membership function and the corresponding linguistic values of the input parameters $\mu_{\mathrm{Y}}, \gamma, \mathrm{r}$.

\begin{tabular}{|l|c|l|}
\hline Input Variables & No. of Membership Functions & Linguistic Values \\
\hline Arrival rate in vacation state $(\lambda)$ & 5 & Very Low; Low; Moderate; High; Very High \\
\hline Service Rate in the busy state $\left(\boldsymbol{\mu}_{\mathrm{Y}}\right)$ & 5 & Very Low; Low; Moderate; High; Very High \\
\hline Repair Rate $(\gamma)$ & 5 & Very Low; Low; Moderate; High; Very High \\
\hline Repair to busy rate $(\mathbf{r})$ & 5 & Very Low; Low; Moderate; High; Very High \\
\hline
\end{tabular}


International Journal of Mathematical, Engineering and Management Sciences

Vol. 6, No. 3, 894-910, 2021

https://doi.org/10.33889/IJMEMS.2021.6.3.053

Table 3. Cost optimization with optimal parameters $\lambda$, $\mu_{\mathrm{Y}}, \beta$, and $\gamma$ for $\mathrm{M} / \mathrm{M} / 1 / \mathrm{N}$ model.

\begin{tabular}{|c|c|c|c|c|c|c|}
\hline & \multicolumn{7}{|c|}{$(\beta, \gamma)$} \\
\hline $\boldsymbol{\lambda}$ & $\mu_{\mathrm{Y}}$ & $\mathbf{( 3 , 5 )}$ & $\mathbf{( 3 . 5 , 5 . 5 )}$ & $\mathbf{( 4 , 6 )}$ & $\mathbf{( 4 . 5 , 6 . 5 )}$ & $\mathbf{( 5 , 7 )}$ \\
\hline & 5 & 350.693 & 407.3237 & 497.7085 & 645.3549 & 884.7603 \\
\hline & 6 & 307.0741 & 325.6668 & 350.7344 & 385.4967 & 435.0186 \\
\hline $\mathbf{0 . 5}$ & 7 & $\mathbf{3 0 0 . 4 2 9 4}$ & 309.4199 & 320.4604 & 334.278 & 351.8978 \\
\hline & 8 & 304.7518 & $\mathbf{3 1 0 . 0 7 2 3}$ & $\mathbf{3 1 6 . 2 5 6 3}$ & $\mathbf{3 2 3 . 5 4 9 5}$ & 332.2666 \\
\hline & 9 & 313.556 & 317.106 & 321.0861 & 325.6056 & $\mathbf{3 3 0 . 7 9 3 8}$ \\
\hline & 10 & 324.57 & 327.132 & 329.932 & 333.0291 & 336.4884 \\
\hline & 5 & 443.0194 & 575.5649 & 801.8198 & 1169.464 & 1699.493 \\
\hline & 6 & 332.8384 & 364.9161 & 411.2044 & 479.9146 & 583.8863 \\
\hline $\mathbf{1}$ & 7 & 311.5135 & 324.6785 & 341.4937 & 363.4523 & 392.7564 \\
\hline & 8 & $\mathbf{3 1 0 . 7 2 4 3}$ & $\mathbf{3 1 7 . 8 2 9 8}$ & $\mathbf{3 2 6 . 2 9 1 2}$ & 336.5313 & 349.1149 \\
\hline & 9 & 317.2332 & 321.7083 & 326.8085 & $\mathbf{3 3 2 . 6 9 9 3}$ & $\mathbf{3 3 9 . 5 8 4}$ \\
\hline & 10 & 327.0407 & 330.146 & 333.5803 & 337.4251 & 341.7732 \\
\hline & 5 & 679.9667 & 1025.401 & 1566.98 & 2257.509 & 2939.965 \\
\hline $\mathbf{1 . 5}$ & 6 & 380.7279 & 443.2999 & 540.522 & 692.9652 & 927.5922 \\
\hline & 7 & 328.6961 & 349.3432 & 377.0725 & 415.2364 & 468.9061 \\
\hline & 8 & $\mathbf{3 1 9 . 0 8 6 2}$ & 328.9677 & 341.0966 & 356.2578 & 375.5456 \\
\hline & 9 & 322.0632 & $\mathbf{3 2 7 . 8 5 4 5}$ & $\mathbf{3 3 4 . 5 8 5 9}$ & $\mathbf{3 4 2 . 5 2 3}$ & 352.0049 \\
\hline & 10 & 330.1478 & 333.9809 & 338.2792 & 343.1598 & $\mathbf{3 4 8 . 7 6 1 7}$ \\
\hline & 5 & 1324.805 & 2064.941 & 2851.716 & 3475.667 & 3881.062 \\
\hline & 6 & 482.1724 & 621.4742 & 846.5063 & 1190.731 & 1664.438 \\
\hline $\mathbf{2}$ & 7 & 357.4153 & 392.8194 & 443.3154 & 516.8357 & 624.9943 \\
\hline & 8 & 331.3264 & 345.7972 & 364.2473 & 388.2554 & 420.0968 \\
\hline & 9 & $\mathbf{3 2 8 . 5 9 3 5}$ & $\mathbf{3 3 6 . 3 3 3 1}$ & 345.548 & 356.6926 & 370.3707 \\
\hline & 10 & 334.1347 & 348.9767 & 344.4813 & 350.8461 & 358.2824 \\
\hline
\end{tabular}

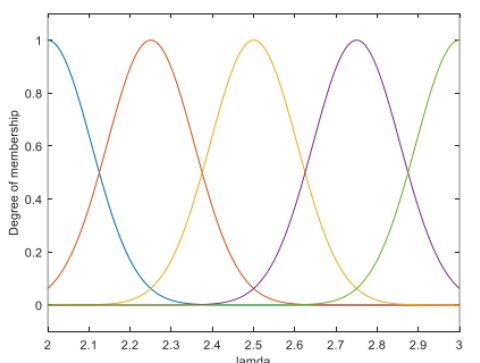

(a)

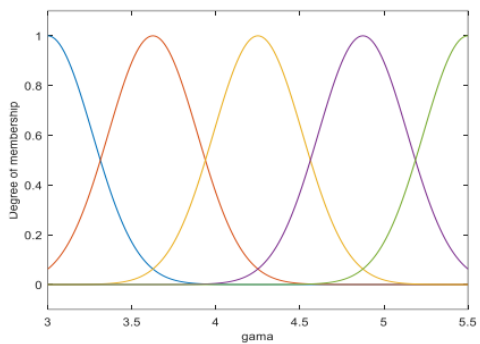

(c)

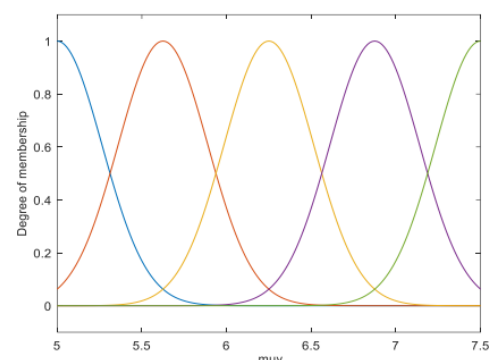

(b)

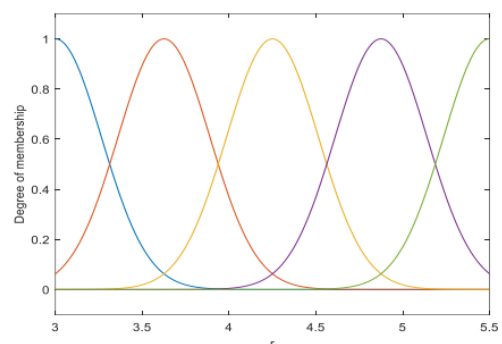

(d)

Figure 3. Membership function for input variables (a) $\lambda$ (b) $\mu_{\mathrm{Y}}$ (c) $\gamma$ and (d) $\mathrm{r}$. 
International Journal of Mathematical, Engineering and Management Sciences

Vol. 6, No. 3, 894-910, 2021

https://doi.org/10.33889/IJMEMS.2021.6.3.053

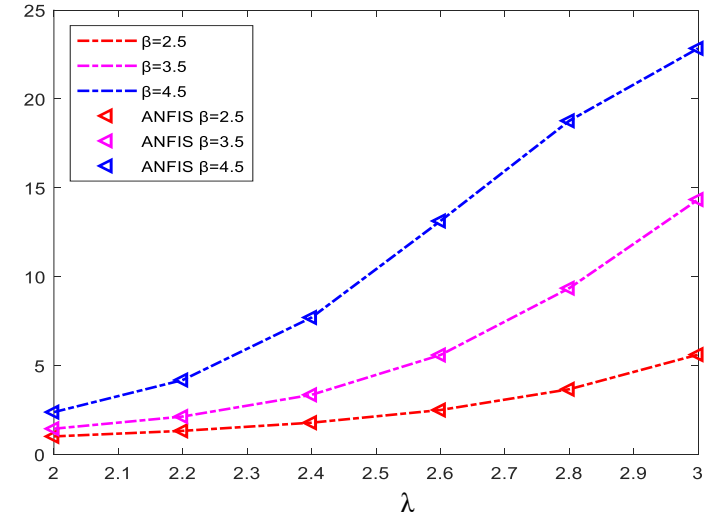

(a)

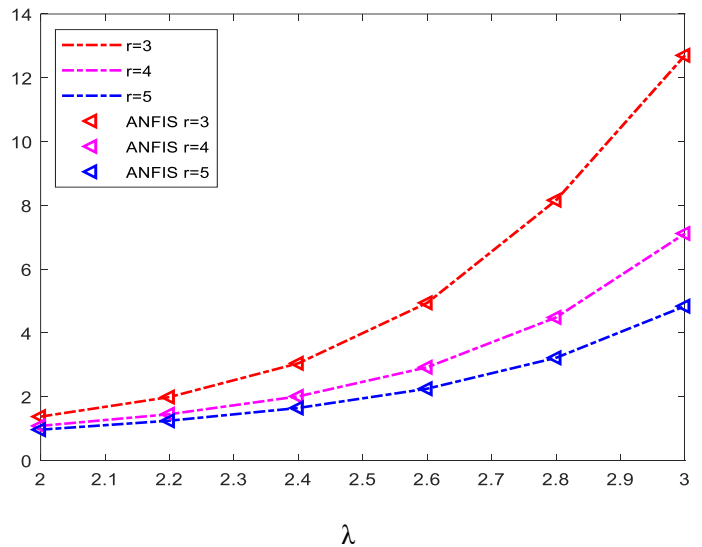

(c)

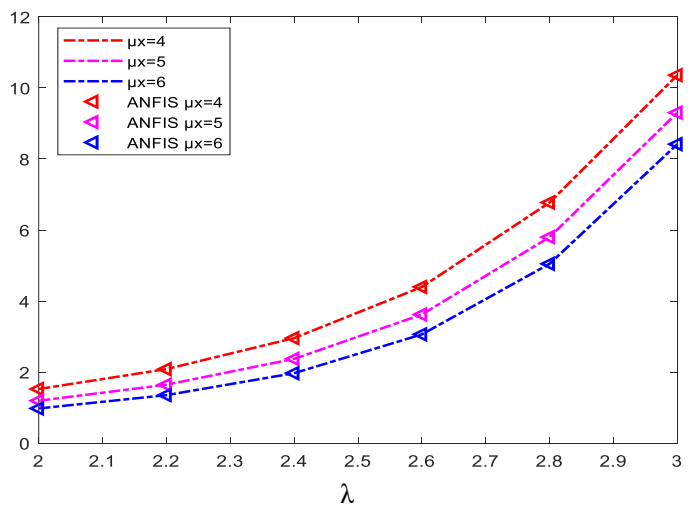

(e)

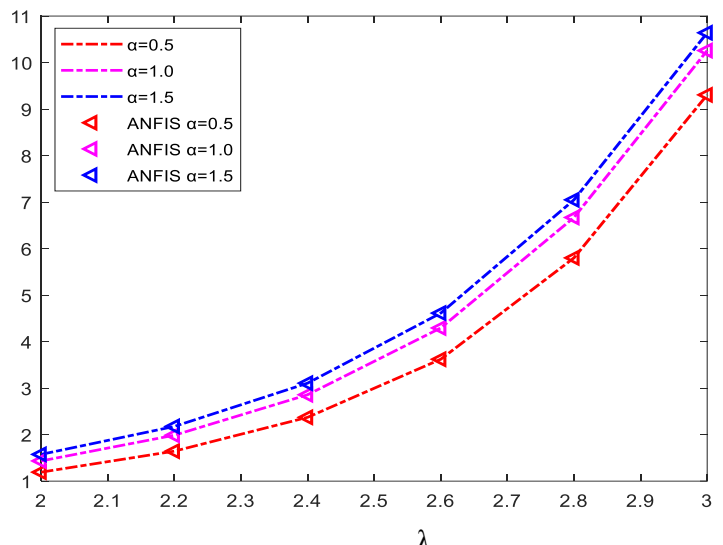

(b)

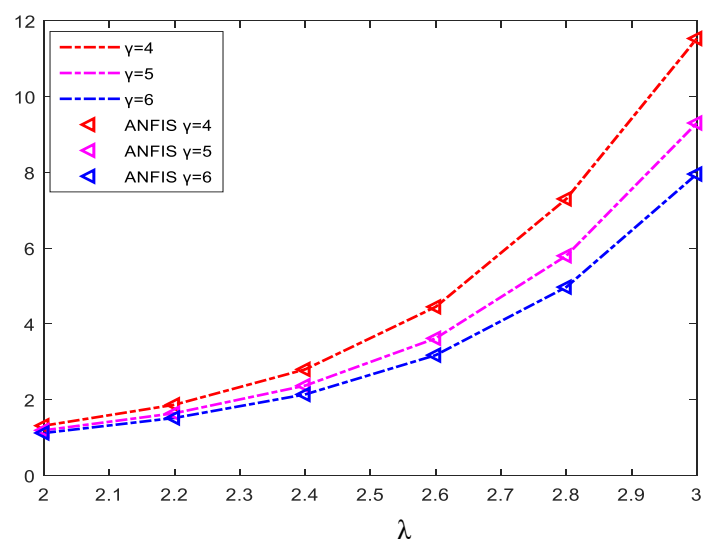

(d)

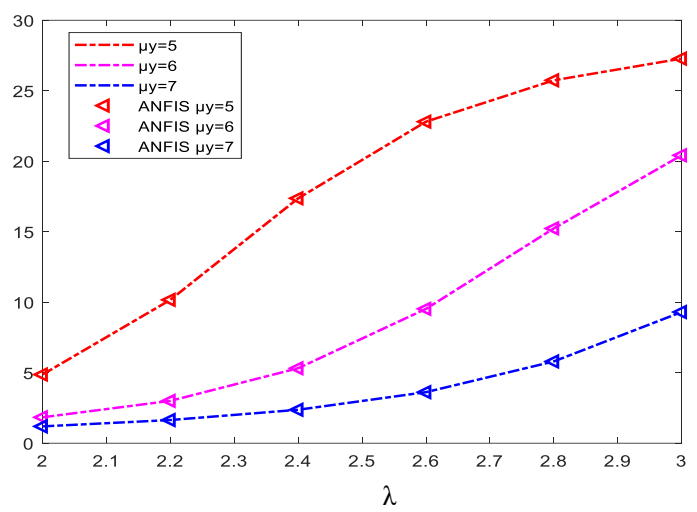

(f)

Figure 4. Effect of $\lambda$ on $E(N)$ with different parameters (a) breakdown rate $(\beta)(b)$ vacation rate $(\alpha)$ (c) repair rate $(\gamma)(\mathrm{d})$ setup rate $(\mathrm{r})$ on ANFIS model (e) operational vacation state $\left(\mu_{\mathrm{X}}\right)(\mathrm{f})$ service rate in busy state $\left(\mu_{Y}\right)$. 
International Journal of Mathematical, Engineering and Management Sciences

Vol. 6, No. 3, 894-910, 2021

https://doi.org/10.33889/IJMEMS.2021.6.3.053

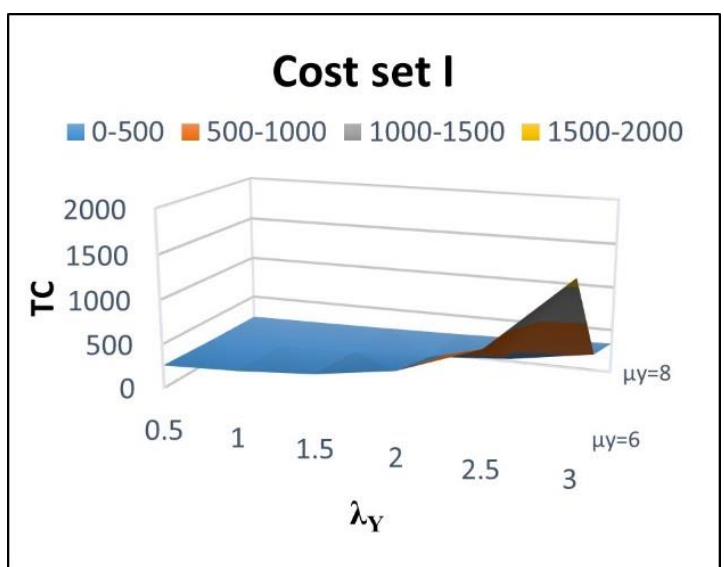

(a)

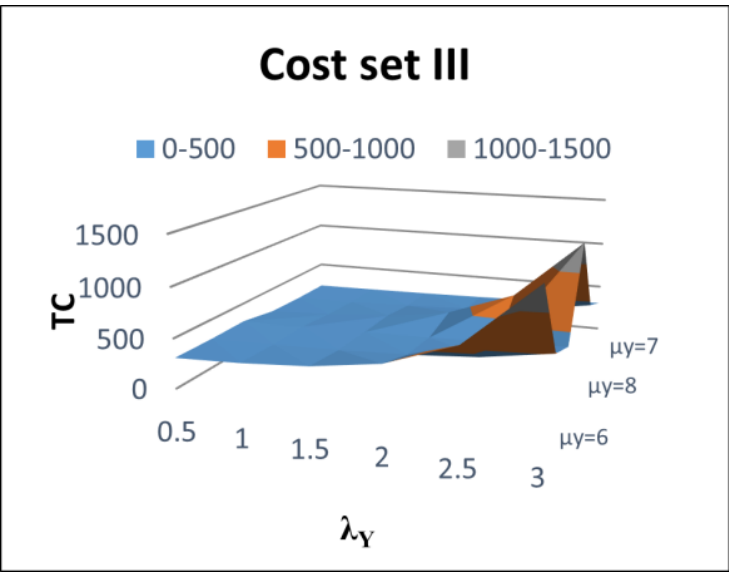

(c)

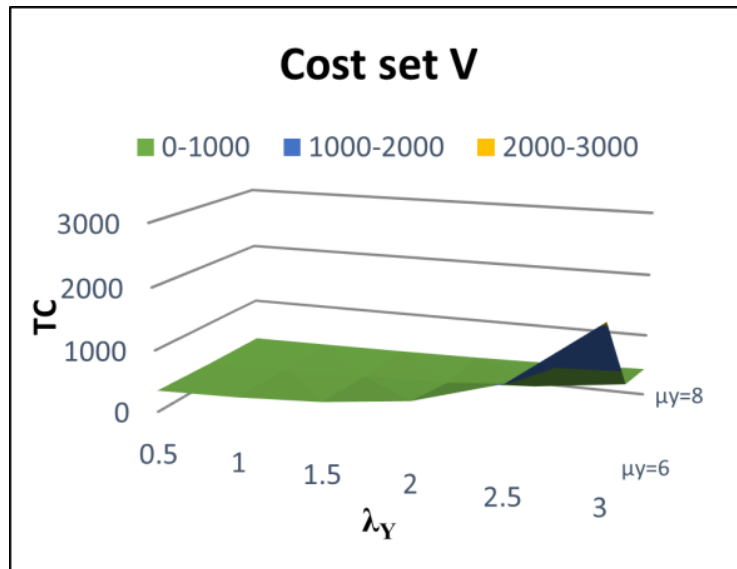

(e)

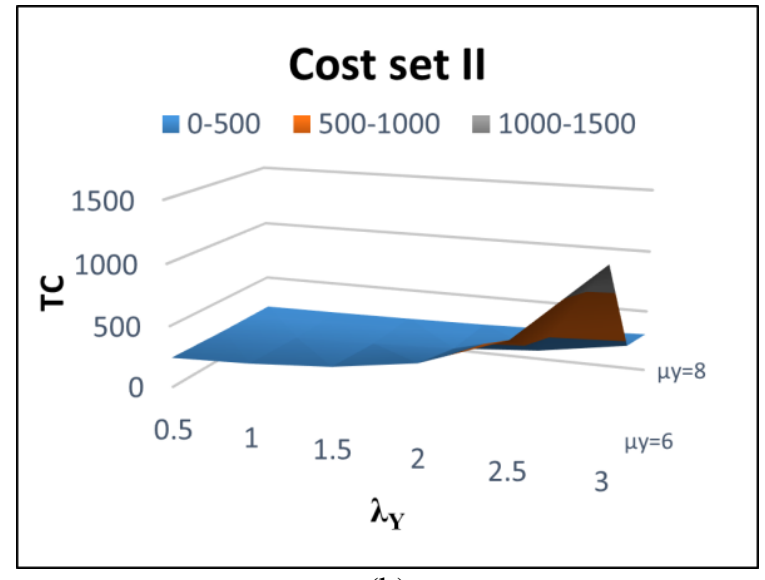

(b)

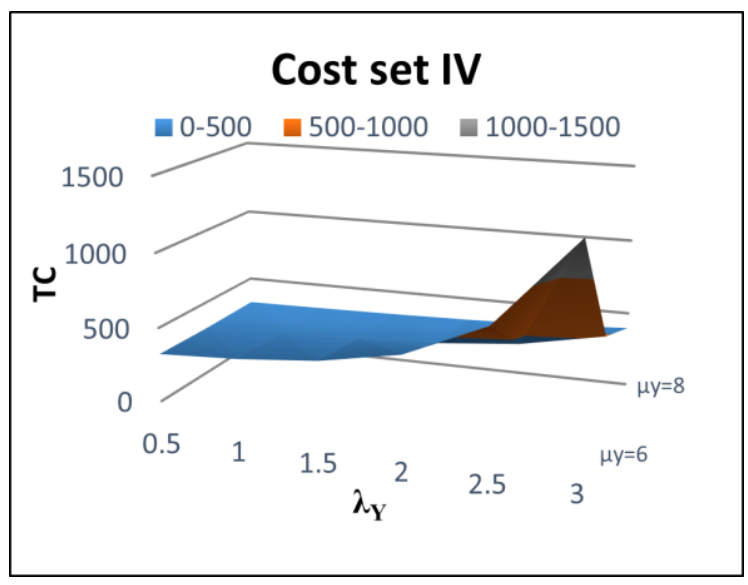

(d)

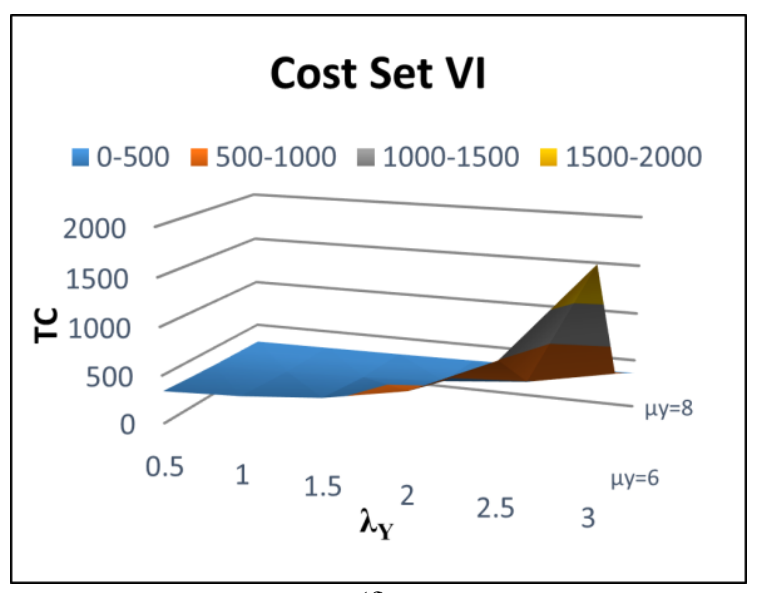

(f)

Figure 5. TC vs. $\left(\mu_{Y}, \lambda_{Y}\right)$ for different cost set (a) Set I (b) Set II (c) Set III (d) Set IV (e) Set V (f) Set VI. 
International Journal of Mathematical, Engineering and Management Sciences

Vol. 6, No. 3, 894-910, 2021

https://doi.org/10.33889/IJMEMS.2021.6.3.053

\section{Conclusions}

This paper deals with the study of a single server Markovian queueing model by considering the realistic feature of operational vacation. The behavior of impatient customers due to vacation is also examined in this study. The situations of server breakdown and repair setup dealt in the model are studied successfully via Matrix geometric method. The sensitivity analysis to know the effect of various parameters on excepted queue length are demonstrated through graphs and tables. The concepts of vacation and the unreliable server incorporated in our model can be observed frequently in daily life including banking sectors, communication centers, power plants, hospitals, call centers, etc. By formulating the cost function, minimum cost has been evaluated to determine the optimal arrival rate and service rate. To make model useful and computable feasible, ANFIS model results are presented which also show the agreements with the matrix geometrical model results. We can further proceed to modify our model by considering the multi-server queueing model operating under F-policy.

\section{Conflict of Interest}

On behalf of all authors, the corresponding author states that there is no conflict of interest.

\section{Acknowledgments}

The authors would like to express their sincere thanks to the editor and anonymous reviewers for their constructive comments and valuable suggestions.

\section{References}

Ahuja, A., Jain, A., \& Jain, M. (2019). Finite population multi-server retrial queueing system with an optional service and balking. International Journal of Computers and Applications, 41(1), 54-61.

Ammar, S.I. (2017). Transient solution of an M/M/1 vacation queue with a waiting server and impatient customers. Journal of the Egyptian Mathematical Society, 25(3), 337-342.

Azhagappan, A., \& Deepa, T. (2020). Variant impatient behavior of a Markovian queue with balking reserved idle time and working vacation. RAIRO Operations Research, 54(3), 783-793.

Bhargava, C., \& Jain, M. (2014). Unreliable multiserver queueing system with modified vacation policy. Opsearch, 51(2), 159-182.

Chakravarthy, S.R., Shruti., \& Kulshrestha, R. (2020). A queueing model with server breakdowns, repairs, vacations, and backup server. Operations Research Perspectives. 7, Article 100131, https://doi.org/10.1016/j.orp.2019.100131.

Chang, F.M., Liu, T.H., \& Ke, J.C. (2018). On an unreliable-server retrial queue with customer feedback and impatience. Applied Mathematical Modelling, 55, 171-182.

Ezeagu, N.J., Orwa, G.O., \& Winckler, M.J. (2018). Transient analysis of a finite capacity $\mathrm{m} / \mathrm{m} / 1$ queuing system with working breakdowns and recovery policies. Global Journal of Pure and Applied Mathematics, 14(8), 1049-1065.

Jain, A., Ahuja, A., \& Jain, M. (2017). Service halt in M/M/1 queue with functioning vacation and customer intolerance. Global and Stochastic Analysis, 4(1), 157-169.

Jain, M., Shekhar, C., \& Shukla, S. (2016). Queueing analysis of machine repair problem with controlled rates and working vacation under F-policy. Proceeding of the National Academy of Sciences, India Section A: Physical Sciences, 86(1), 21-31.

Jeyakumar, S., \& Senthilnathan, B. (2012). A study on the behavior of the server breakdown without interruption in a $\mathrm{M}^{\mathrm{x}} / \mathrm{G}(\mathrm{a}, \mathrm{b}) / 1$ queueing system with multiple vacations and closedown time. Applied Mathematics and Computation, 219(5), 2618-2633. 
International Journal of Mathematical, Engineering and Management Sciences

Vol. 6, No. 3, 894-910, 2021

https://doi.org/10.33889/IJMEMS.2021.6.3.053

Kalyanaraman, R., \& Sundaramoorthy, A. (2019). A Markovian working vacation queue with server statedependent arrival rate and partial breakdown. International Journal of Recent Technology and Engineering, 7(6), 664-668.

Jain, M., \& Meena, R.K. (2018). Vacation model for Markov machine repair problem with two heterogeneous unreliable servers and threshold recovery. Journal of Industrial Engineering International, 14(1), 143152.

Neuts, M.F. (1981). Matrix geometric solutions in stochastic models: An Algorithmic Approach. The John Hopkins University $\quad$ Press, $\quad$ Baltimore, $\quad 1981, \quad 352$, https://onlinelibrary.wiley.com/doi/abs/10.1002/net.3230130219.

Niranjan, S.P., Chandrasekaran, V.M., \& Indhira, K. (2017). Queue size-dependent service in bulk arrival queueing system with server loss and vacation break-off. International Journal of Knowledge Management in Tourism and Hospitality, 1(2), 176-207.

Raj, M.R., \& Chandrasekar, B. (2016). Matrix-Geometric method for queueing model with system breakdown, standby server, ph service and ph repair. International Journal of Mathematics Research, 8(1), 29-37.

Rao, S.H., Kumar, V.V., Kumar, B.S., \& Rao, T.S. (2017). Analysis of two- phase queuing system with impatient customers, server breakdown and delayed repair. International Journal of Pure and Applied Mathematics, 115(4), 651-663.

Sanga, S.S., \& Jain, M. (2019). Cost optimization and ANFIS computing for admission control of M/M/1/K queue with general retrial times and discouragement. Applied Mathematics and Computation, Elsevier, 363, https://doi.org/10.1016/j.amc.2019.124624.

Sethi, R., Jain, M., Meena, R.K., \& Garg, D. (2020). Cost optimization and ANFIS computing of an unreliable $\mathrm{M} / \mathrm{M} / 1$ queueing system with customers' impatience under n-policy. International Journal of Applied and Computational Mathematics, 6(2). doi:10.1007/s40819-020-0802-0.

Tian, R., \& Wang, Y. (2020). Optimal strategies and pricing analysis in M/M/1 queues with a single working vacation and multiple vacations. RAIRO Operation Research, 54(6), 1593-1612.

Upadhyaya, S., \& Kushwaha, C. (2020). Performance prediction and ANFIS computing for unreliable retrial queue with delayed repair under modified vacation policy. International Journal of Mathematics in Operational Research, 17(4), 437-466.

Vijayalakshmi, V., Kalidass, K., \& Pavitha, K. (2018). An M/M/1/N queue with working breakdowns and a two-phase service. International Journal of Pure and Applied Mathematics, 119(15), 2285-2297.

Ye, Q., \& Liu, L. (2017). Analysis of MAP/M/1 queue with working breakdowns. Communications in Statistics-Theory and Methods, 47(13), 3073-3084.

Original content of this work is copyright $\odot$ International Journal of Mathematical, Engineering and Management Sciences. Uses under the Creative Commons Attribution 4.0 International (CC BY 4.0) license at https://creativecommons.org/licenses/by/4.0/ 